\title{
Evaluación económica de la prueba genética de la poliposis adenomatosa familiar
}

\author{
A. Olry de Labry Lima ${ }^{1,2}$, L. Sordo del Castillo ${ }^{3}$, L. García Mochón $^{1}$, D. Epstein ${ }^{4}$, C. Bermúdez Tamayo ${ }^{1}$ y \\ R. Villegas Portero ${ }^{5}$
}

${ }^{1}$ Escuela Andaluza de Salud Pública. Granada. ${ }^{2}$ CIBER en Epidemiología y Salud Pública (CIBERESP). ${ }^{3}$ Servicio de Medicina Preventiva. Hospital Universitario Virgen de las Nieves. Granada. ${ }^{4}$ Centre for Health Economics. University of York. ${ }^{5}$ Agencia de Evaluación de Tecnologías Sanitarias de Andalucía. Sevilla

\section{RESUMEN}

Objetivo: analizar el coste-utilidad de la prueba genética a familiares de primer grado de pacientes con cáncer de colon para determinar mutaciones del gen APC (Adenomatous Polyposis Coli).

Metodología: los análisis se realizaron desde el punto de vista del sistema sanitario. Se utilizó un modelo de Markov. Realización de la prueba genética para el gen APC, causante de la poliposis adenomatosa familiar (PAF), que produce cáncer de colon frente a la no realización de la misma. La medida de efectividad utilizada fueron los años de vida ajustados por calidad (AVAC) y la unidad de coste los euros de 2005. Los costes de las intervenciones fueron extraídos de los precios públicos de los servicios sanitarios prestados por centros dependientes del Sistema Sanitario Público Andaluz y los valores de la efectividad y de utilidad de la literatura.

Resultados: la realización de la prueba genética se muestra como una estrategia dominante a la no realización de la misma, ya que esta última tiene un coste incremental de 7.676,34 €, además de una menor efectividad. Los análisis de sensibilidad mostraron que la realización de la prueba genética se mantiene como la estrategia dominante dentro de un amplio rango de coste de la prueba y de probabilidad de desarrollar adenocarcinomas.

Conclusiones: los análisis mostraron que, para este grupo de pacientes, la realización de la prueba genética para la detección de la mutación del gen APC es en promedio menos costosa y además produce una mejora en AVAC comparado con la no realización de la misma.

Palabras clave: Poliposis adenomatosa familiar. Gen APC. Cáncer de colon.

\begin{abstract}
Objective: to analyze the cost-effectiveness of genetic testing for first-degree relatives of patients with colon cancer to identify mutations in the APC gene (Adenomatous Polyposis Coli).

Methodology: analyses were performed from the perspective of the health system. We used a Markov model. We compared genetic testing for the APC gene, the cause of familial adenomatous polyposis (FAP), which results in colon cancer, versus no genetic testing for said gene. The effectiveness measure used was quality-adjusted life-years (QALYs), and costs were measured in euros for 2005. The costs of interventions were extracted from the costs of health services provided by centers under the Andalusian Public Health System, and other parameters were obtained from the literature.

Results: the performance of genetic testing is the dominant strategy when compared to the absence of genetic testing given the latter option has an incremental cost of $€ 7,676.34$ and is less effective. A sensitivity analysis found that genetic testing remains the dominant strategy for a plausible range of costs of the test itself, and for the probability of developing adenocarcinoma.

Conclusions: our analysis showed that in this patient group genetic testing to detect APC gene mutations is on average less costly and improves QALYs versus no testing.
\end{abstract}

Key words: Familial adenomatous polyposis. APC gene. Colon cancer.

Olry de Labry Lima A, Sordo del Castillo L, García Mochón L, Epstein D, Bermúdez Tamayo C, Villegas Portero R. Evaluación económica de la prueba de la poliposis adenomatosa familiar. Rev Esp Enferm Dig 2008; 100: 470-475.

Recibido: 21-04-08

Aceptado: $17-06-08$

Este trabajo se ha realizado bajo la dirección técnica de la Agencia de Evaluación de Tecnologías Sanitarias de Andalucía (AETSA) en el marco de colaboración previsto en el Plan de Calidad para el Sistema Nacional de Salud, al amparo del convenio de colaboración suscrito por el Instituto de Salud Carlos III, organismo autónomo del Ministerio de Sanidad y Consumo y la Fundación Progreso y Salud de Andalucía.

Correspondencia: Antonio Olry de Labry Lima. Escuela Andaluza de Salud Pública. Campus Universitario de Cartuja. Apdo. correos 2070. 18080 Granada. e-mail: antonio.olrylabry.easp@juntadeandalucia.es

\section{INTRODUCCIÓN}

En los últimos años han sido descritas mutaciones genéticas hereditarias relacionadas con algunas enfermedades y que podrían permitir identificar a sujetos con alto riesgo de padecer alguna de ellas a lo largo de su vida. En este sentido, el análisis genético ofrece la oportunidad de un diagnóstico precoz que permita establecer medidas preventivas y terapéuticas precoces para mejorar la supervivencia de estos pacientes (1). 
Dentro de las variantes hereditarias del cáncer de colon se cuentan dos síndromes en los cuales la predisposición a la enfermedad se basa en una mutación de la línea germinal (2). Uno de ellos es la poliposis adenomatosa familiar (PAF), con una tasa de incidencia de $1 / 10.000$ y causante de alrededor del $1-2 \%$ de los cánceres colorrectales $(3,4)$.

La PAF parece ser causada por mutaciones del gen APC y se transmite en forma autosómica dominante con una penetrancia de $100 \%(3,4)$. La PAF se manifiesta primariamente como múltiples pólipos $(>100)$ de aparición temprana, con tamaño variado y se asocia a lesiones en otros parénquimas $(3,4)$. La degeneración carcinomatosa de una o más de estas formaciones es considerada inevitable si se deja sin tratamiento (57).

Con anterioridad a la implantación del estudio genético, a todos los familiares de personas diagnosticadas de PAF se les realizaba un seguimiento colonoscópico. Actualmente, el análisis genético de APC puede permitir la identificación presintomática de aquellos familiares en riesgo. Ello hace que aquellos familiares en los que no se determina la presencia del gen APC queden excluidos de la realización de colonoscopias, permitiendo que el seguimiento se centre sólo en aquellos portadores de la mutación (3).

La investigación de resultados en salud se divide en tres áreas principales: resultados clínicos, económicos y humanísticos. Los resultados clínicos tratan con temas de eficacia o efectividad de la intervención en el contexto de los servicios sanitarios. Los resultados económicos tratan del coste y la utilización de recursos por las intervenciones en salud relacionados con la obtención de la eficacia clínica, y los resultados humanísticos son específicos al paciente y enfocados a la calidad de vida obtenida con cada intervención, al igual que con resultados que el paciente reporta como son síntomas físicos, psicológicos y de morbilidad. Todo ello permite a los análisis de evaluación económica combinar el conocimiento de la medicina, estadística, economía y la epidemiología, y acercar el método científico en la toma de decisiones basada en evidencia. En cuanto al análisis de cribado del cáncer colorrectal, permite seleccionar al programa o seguimiento específico más idóneo para esta población susceptible $(8,9)$.

Por otro lado, cabe destacar que estudios previos han puesto de manifiesto que la realización de la prueba genética es menos costosa que la colonoscopia. Sin embargo, ninguno de ellos tuvo en cuenta los riesgos de morbimortalidad de la colonoscopia, ni se contempló bajo las nuevas técnicas de detección de mutaciones genéticas (10-12). Por todo ello el objetivo de este trabajo es realizar una evaluación económica de la prueba genética para el gen APC (Adenomatous Polyposis Coli), causante de la poliposis adenomatosa familiar (PAF) que cursa con cáncer de colon.

\section{MÉTODOS}

Se realizó una simulación a través de un modelo de Markov, que permite hacer simulaciones de procesos sanitarios complejos. Estos análisis se realizaron bajo la perspectiva del sistema sanitario. Para ello se partió de una cohorte hipotética de adolescentes de 14 años, con un familiar de primer grado diagnosticado de PAF, a los que está indicada una especial vigilancia (realización de prueba genética y/o colonoscopia anual). Se consideraron tres estados de salud (Fig. 1); así, todos los pacientes serían inicialmente "asintomáticos" y, con el tiempo, irían transitando (debido a la progresión de la enfermedad) a los estados "sintomáticos" y "muerte". Las transiciones entre los estados se harían en unos periodos discretos de tiempo denominados "ciclos", con una duración de 1 año, utilizándose como horizonte temporal la esperanza de vida de los pacientes.

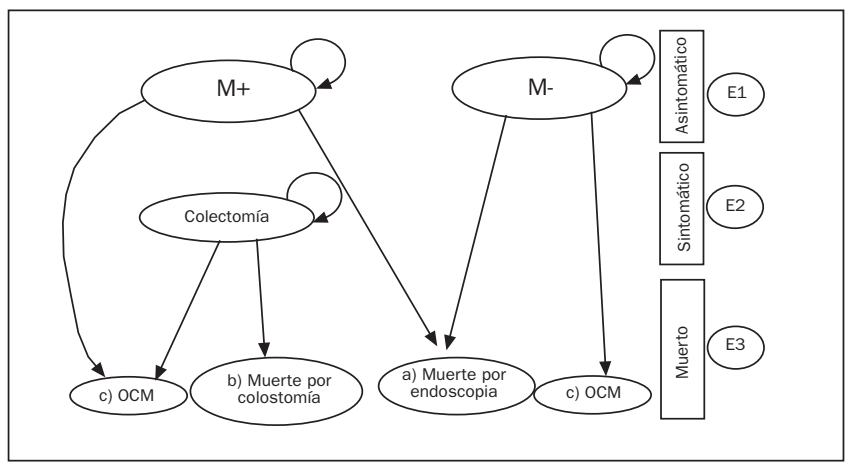

Fig. 1. Estructura del diagrama del modelo de Markov utilizado para pacientes a los que se les realiza la prueba genética (OCM: otras causas de muerte; $M+$ : pacientes portadores de la mutación del gen APC; $M$-: pacientes no portadores de la mutación del gen APC. La estructura del modelo para pacientes a los que no se les realiza la prueba es similar, aunque debido a que su estatus genético es desconocido, todos los pacientes asintomáticos reciben una endoscopia anual).

Las estrategias que se compararon fueron el seguimiento de familiares con y sin cribado genético. En la figura 1 se pueden observar los tres estados de salud utilizados en el modelo: a) asintomático: sujetos no portadores y portadores asintomáticos; b) sintomáticos: pacientes que han desarrollado la enfermedad y han sido sometidos a colectomía; y c) muerte. Esta tiene tres causas diferentes: a) debido a la realización de la colonoscopia; b) debido a la operación de colectomía; y c) otras causas de muerte (no relacionadas con la patología objeto de estudio).

Cada estado de salud tiene asociadas unas probabilidades de transición, unas utilidades y unos costes. Los resultados en salud se midieron como años de vida ajustados por calidad (AVAC), siendo un AVAC un año de vida multiplicado por un factor de ponderación que 
indica la calidad de vida de la persona durante ese año. El factor de ponderación de la calidad de un año de vida puede ir desde el valor 0 (muerte o equivalente), hasta el 1 (salud perfecta). Las utilidades consideradas en el modelo se obtuvieron a partir de varios estudios previos $(13,14)$.

El seguimiento anual mediante colonoscopia está indicado tanto en aquellos sujetos a los que no se les realiza el test genético, como en los que tienen un test positivo. Una vez que el paciente portador de la mutación presenta un número determinado de adenocarcinomas, acaba sometiéndose a colectomía para eliminarlos (Fig. 2). En aquellos pacientes con prueba genética negativa están indicadas cuatro colonoscopias $(18,25,35$ y 50 años) $(2,15)$. En el caso de no realizarse el test genético, se considera que el paciente no es portador de la enfermedad cuando a los 50 años se mantiene como asintomático (1). Todos los pacientes, independientemente de la realización del test genético, pueden fallecer por otras causas de muerte (OCM) durante el seguimiento (8).

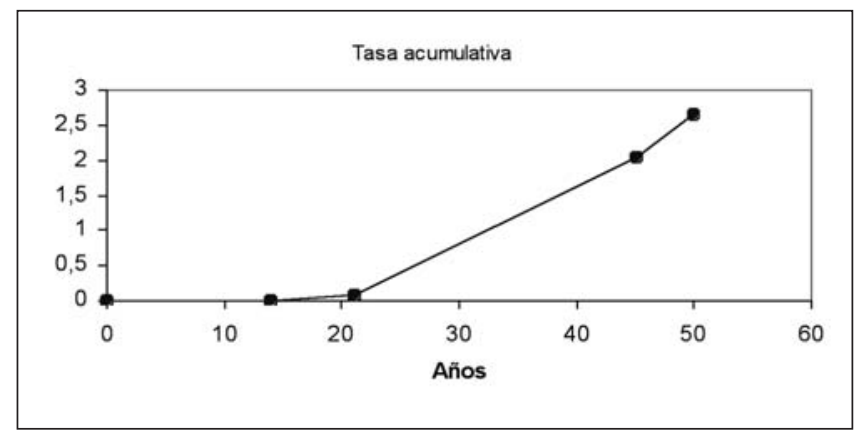

Fig. 2. Tasa acumulada de desarrollar adenocarcinomas para los pacientes portadores de la mutación.

\section{Efectividad y asunciones del modelo de Markov}

Se consideró una penetrancia de la mutación del APC del $100 \%$ (4). El cálculo de la probabilidad anual de desarrollar adenocarcinomas susceptibles de colectomía se realizó a partir del estudio de Bussey (1975) (16). Este trabajo recoge que el $7 \%$ de los pacientes no tratados desarrolla cáncer a los 21 años, mientras que el 87 y $93 \%$ lo desarrollan a los 45 y 50 años respectivamente. Con estos datos se obtuvieron unas probabilidades anuales entre los 0 y 21 años del 3,39 y del 8,53\% hasta el final (Fig. 1).

Las complicaciones relacionadas con la colectomía y colonoscopia se tuvieron en cuenta para la imputación de costes y la mortalidad (17-19). Por el contrario, cuando se realiza el test genético y este es negativo, se repite el análisis genético; este aspecto también se considera para la imputación de costes. Se consideró que la prueba de detección de mutaciones fue la secuenciación con una sensibilidad y especificidad del $100 \%$. Se asignaron los siguientes valores de utilidad: pacientes operados de colectomía 0,5 (asunción de los autores), asintomático-saben que tienen adenomas 0,91 (13) y 0 para la muerte.

En la tabla I se pueden observar los costes utilizados. Se asumió que el paciente era atendido en las urgencias de un centro de atención primaria que precisaba observación y posterior derivación a un centro hospitalario y que este transporte de emergencia se realizaba en UVI móvil terrestre en zona urbana.

Tabla I. Costes y probabilidades utilizadas en el modelo de Markov

\begin{tabular}{lc}
\hline Coste de las intervenciones y pruebas $(€)$ & \\
\hline Prueba genética & $1.164,9 €$ \\
Colonoscopia & $121,72 €$ \\
Perforación durante colonoscopia & $3.065,25 €$ \\
Colonoscopia ponderada & $734,77 €$ \\
Colectomía con complicaciones & $11.720,55 €$ \\
Colectomía sin complicaciones & $7.484,75 €$ \\
Colectomía ponderada & $9.009,63 €$ \\
Coste de la muerte & $837,86 €$ \\
\hline Efectividad y asunciones del modelo de Markov & \\
\hline Probabilidad anual de desarrollar adenocarcinomas (6) & \\
0-21 años & $3,39 \%$ \\
> 21 años & $8,53 \%$ \\
CC de colectomía (8) & $36 \%$ \\
Mortalidad por colectomía (8) & $0,7 \%$ \\
Probabilidad de heredar mutación & $50 \%$ \\
Mortalidad por colonoscopia (9,10) & $0,01 \%$ \\
Perforación durante colonoscopia (9,10) & $0,2 \%$ \\
\hline
\end{tabular}

Los costes de los diferentes procedimientos se obtuvieron a partir de los grupos relacionados con el diagnostico (GRD) según los precios públicos de los servicios sanitarios prestados por centros dependientes del Sistema Sanitario Público Andaluz (20) y se presentan en euros de 2005. La mortalidad por otras causas de muerte para los diferentes tramos de edad se obtuvo del Instituto Nacional de Estadística (INE) (21).

Los resultados se expresan como el coste por AVAC incremental, que es el ratio entre la diferencia de los costes y AVAC promedio entre las alternativas consideradas. Una estrategia resulta dominante cuando presenta un menor coste y una mayor efectividad que la otra estrategia. La tasa de descuento utilizada fue del 3,5\%, tanto para los costes como para los resultados en salud.

Finalmente, para establecer la robustez del modelo y explorar las diferencias entre las opciones se realizaron análisis de sensibilidad de una y dos vías, modificando el coste de la realización de la prueba genética en un 50\%, la probabilidad anual constante de desarrollar adenocarcinoma a un $0,2 \%$ anual y la prevalencia de pacientes 
portadores de la mutación (25-75\%). Para la elaboración del modelo de Markov y el análisis de los datos se utilizó el programa Tree-Age 2007.

\section{RESULTADOS}

El coste promedio de la estrategia de control de la enfermedad mediante la prueba genética fue 8.038,93€, frente al de 13.928,82€ cuando la vigilancia de la enfermedad no incluye análisis genético y se realiza mediante colonoscopia anual. Se observa que la alternativa de no aplicar el test resulta dominada por la realización de la prueba, ya que implica un incremento de coste de $5.889,89 €$ y una pérdida de 0,01 de AVAC con respecto a la aplicación del test genético (Tabla II).

Tabla II. Análisis coste-utilidad de la prueba de detección de la mutación del gen APC causante del cáncer de colon

\begin{tabular}{lcccccc}
\hline & Coste & $\begin{array}{c}\text { Coste Efectividad } \\
\text { incremental }\end{array}$ & $\begin{array}{c}\text { Efectividad } \\
\text { incremental }\end{array}$ & $\begin{array}{c}\text { Coste } \\
\text { utilidad }\end{array}$ & $\begin{array}{c}\text { Coste/utilidad } \\
\text { incremental }\end{array}$ \\
\hline Test APC & $8.038,93 €$ & & 19,93 & & 403,15 & \\
No test APC & $13.928,82 €$ & $5.889,89 €$ & 19,92 & $-0,01$ & 699,18 & Dominado \\
\hline
\end{tabular}

Se realizó un análisis de sensibilidad modificando el coste de la realización de la prueba genética, la probabilidad de desarrollar adenocarcinoma y la prevalencia de la mutación, estableciendo diferentes combinaciones con ambos parámetros. Así, en todos los casos analizados, la aplicación del test genético resultó ser la alternativa dominante, ya que es la opción de menor coste y mayor efectividad que la no realización de la prueba genética. La no aplicación del test genera un incremento del coste que oscila entre 9.417,29€, si se asume que el $25 \%$ de los pacientes son portadores de la mutación, y 1.634,43€, cuando se aumenta el coste de realización de la prueba en un 50\% y además se supone que el $75 \%$ de los pacientes posee la alteración genética. En cuanto a la efectividad incremental, en cualquiera de los supuestos analizados, se obtiene una pérdida en los AVAC por la no aplicación de la prueba, siendo esta pérdida mayor $(0,02$ de AVAC) si se considera que el $25 \%$ de la población es portadora de la mutación (Tabla III).

\section{DISCUSIÓN}

Los resultados de este estudio ponen de manifiesto que la realización de la prueba genética para la detección de la mutación en el gen APC en el cribado y seguimiento de la PAF resulta dominante (más eficaz y
Tabla III. Análisis de sensibilidad

\begin{tabular}{|c|c|c|c|}
\hline & $\begin{array}{c}\text { Coste } \\
\text { incremental }\end{array}$ & $\begin{array}{l}\text { Efectividad } \\
\text { incremental }\end{array}$ & $\begin{array}{l}\text { Coste/efectividad } \\
\text { incremental }\end{array}$ \\
\hline \multicolumn{4}{|c|}{ Aumento del $50 \%$ en el coste de la realización de la prueba genética } \\
\hline \multicolumn{4}{|l|}{ Test APC } \\
\hline No test $A P C$ & $5.016,22 €$ & $-0,01$ & Dominado \\
\hline \multicolumn{4}{|c|}{ Probabilidad anual constante del 0,2\% de desarrollar adenocarcinoma } \\
\hline \multicolumn{4}{|l|}{ Test APC } \\
\hline No test $A P C$ & $5.889,89 €$ & $-0,01$ & Dominado \\
\hline \multicolumn{4}{|c|}{ Aumento al $75 \%$ de pacientes portadores de la mutación } \\
\hline \multicolumn{4}{|l|}{ Test APC } \\
\hline No test $A P C$ & $2.362,49 €$ & $-0,01$ & Dominado \\
\hline \multicolumn{4}{|c|}{ 25\% de pacientes portadores de la mutación } \\
\hline \multicolumn{4}{|l|}{ Test APC } \\
\hline No test APC & $9.417,29 €$ & $-0,02$ & Dominado \\
\hline \multicolumn{4}{|c|}{$\begin{array}{c}\text { Probabilidad anual al 0,2\% de desarrollar adenocarcinoma y aumento del } 50 \% \text { en el coste de } \\
\qquad \text { la realización de la prueba genética }\end{array}$} \\
\hline \multicolumn{4}{|l|}{ Test APC } \\
\hline No test APC & $5.016,22 €$ & $-0,01$ & Dominado \\
\hline \multicolumn{4}{|c|}{$\begin{array}{l}\text { Aumento del } 50 \% \text { en el coste de la realización de la prueba genética y el } 75 \% \text { de pacientes } \\
\text { portadores de la mutación }\end{array}$} \\
\hline \multicolumn{4}{|l|}{ Test APC } \\
\hline No test APC & $1.634,43 €$ & $-0,01$ & Dominado \\
\hline
\end{tabular}

menores costes) a la realizada mediante seguimiento por colonoscopia. Este resultado ya había sido descrito previamente en otros estudios, pero estos utilizaban como metodología la minimización de costes, la cual asume igual efectividad entre las diferentes alternativas y, por consiguiente, únicamente tiene en cuenta los costes. Así, este es el primer estudio que utiliza una simulación a largo plazo e incluye costes de morbimortalidad y los años de vida ajustados por calidad (1012). Por otro lado, el método de análisis genético aquí analizado es más novedoso que en los estudios previos, que utilizan el método de la proteína truncada $(10-12)$. Estos dos aspectos podrían hacer cambiar los resultados.

Este artículo tiene una serie de limitaciones que deben tenerse en cuenta para la interpretación de los resultados. En primer lugar, no se consideró la ocupación y saturación de los servicios sanitarios. Esta podría verse modificada en dos direcciones: por un lado, se produciría un aumento en la frecuentación de consultas de consejo genético (22); y por otro, la aplicación de la prueba genética haría disminuir el número de colonoscopias, al quedar excluidos de ellas aquellos pacientes sin mutación en el gen APC. En cualquier caso, el cribado genético se realizaría una sola vez, mientras las colonoscopias, que evitan un resultado negativo a dicho test, deberían ser anuales $(23,24)$. En este sentido, es de resaltar que existen referencias en la literatura sobre la dificultad que tendrían los sistemas sanitarios de 
asumir la carga de trabajo que supondría la realización de colonoscopias a todas aquellas personas con riesgo de desarrollar cáncer colorrectal. Así, la implementación del cribado genético del APC es una de las medidas que podría paliar esta dificultad $(14,24)$.

Por otro lado, una revisión sistemática sobre las pruebas genéticas puso de manifiesto que los factores más influyentes sobre el ratio coste-efectividad son la prevalencia de la mutación, el coste del test genético, mortalidad y efectividad del tratamiento (25). Para que quedaran contempladas posibles variaciones en estos parámetros, los análisis de sensibilidad han contemplado un amplio rango de valores en estos factores para determinar la robustez de los resultados y, dada la robustez de los resultados obtenidos, se descartó la posibilidad de realizar un análisis de sensibilidad probabilístico. Por último, las probabilidades utilizadas para reflejar las otras causas de muerte se obtuvieron del Instituto Nacional de Estadística (INE) de 2004 (21). Este hecho puede introducir un sesgo, ya que estas probabilidades podrían cambiar con los años.

Por otro lado, en este estudio no se ha contemplado la posibilidad de que un paciente no acuda al seguimiento. En este sentido, se ha puesto de manifiesto que, aunque la participación de los individuos que tienen un familiar con historial de cáncer colorrectal es mayor que la de aquellos que no lo tienen, el cumplimiento en el seguimiento de las colonoscopias de los pacientes que se han sometido al test genético y son portadores es mayor (73-100\%) que el de aquellos que no se someten al test genético (60\%) (26). Por ello, si se hubiera tenido en cuenta este hecho, se pondría más en evidencia el beneficio tanto en términos de salud como en costes de la realización del test genético.

Los análisis se han realizado desde la perspectiva del sistema sanitario, por ello no se han considerado los costes indirectos de los pacientes o posibles acompañantes, como la pérdida de productividad derivada de la realización de la prueba o a causa de la enfermedad. En cualquier caso, de todos estos costes estarían libres los pacientes no portadores bajo la estrategia del test genético y, de haber considerado los costes indirectos causados por la enfermedad, sólo habría reforzado aún más los resultados encontrados (8).

La utilización de la prueba genética elimina la necesidad de seguimiento anual mediante colonoscopias y sigmoidoscopias a todos los pacientes y sujetos en riesgo en los que no se encontró la mutación familiar. Otro beneficio del test genético es que mejora sustancialmente la prevención del cáncer colorrectal, al conseguir que pacientes que no querían someterse a colonoscopias periódicas aceptaran el análisis molecular, motivando además al resto de familiares reacios a ser estudiados $(25,26)$. Otro aspecto que debería valorarse es que quizás estos pacientes podrían entrar de una forma más directa en el circuito asistencial.

Los pacientes no portadores de la mutación tendrán la certeza de no padecer la enfermedad y no trasmitirla en ningún caso a su futura descendencia, con el consiguiente alivio psicológico, así como la eliminación de numerosas consultas y exploraciones médicas, con sus correspondientes costes sociales y sanitarios (26). Mientras que los pacientes portadores de la mutación requieren un estrecho seguimiento de neoplasias extracolónicas y otras manifestaciones, así la existencia de estos otros factores puede afectar a la estrategia más conveniente para la elección de cribado. Además se deben tener en cuenta las consecuencias del inadecuado uso de estas técnicas en la calidad de vida de los pacientes. Todo ello puede influenciar el cumplimiento del seguimiento del cribado por parte de los pacientes (27). Por último, cabe destacar que los resultados basados en coste por AVAC permiten la comparación con otras tecnologías sanitarias y ayudan en la toma de decisiones a los gestores sanitarios (8).

\section{AGRADECIMIENTOS}

A Pablo Vega, médico del Hospital Virgen de las Nieves (Granada), por su ayuda en los aspectos clínicos.

\section{BIBLIOGRAFÍA}

1. Cruz-Bustillo C. Genética molecular del cáncer colorrectal. Rev Esp Enferm Dig 2004; 96: 48-59.

2. Wong N, Lasko D, Rabelo, R. Genetic counselling and interpretation of genetic test in familial adenomatous polyposis and hereditary nonpolyposis colorectal cancer. Dis Colon Rectum 2001; 44: 271-9.

3. Grupo de trabajo de la guía de práctica clínica de prevención del cáncer colorrectal. Guía de práctica clínica. Barcelona: Asociación Española de Gastroenterología, Sociedad Española de Medicina de Familia y Comunitaria y Centro Cochrane Iberoamericano; 2004. Programa de Elaboración de Guías de Práctica Clínica en Enfermedades Digestivas, desde la Atención Primaria a la Especializada: 4.

4. Fearnhead S, Britton M, Bodmer W. The ABC of APC. Hum Mol Genet 2001; 10: 721-33.

5. Weizt J, Koch M, Debus J, Höhler T, Galle PR, Büchler MW. Colorrectal cancer. Lancet 2005; 365: 153-65.

6. Fearnhead NS, Wilding JL, Bodmer WF. Genetics of colorectal cancer: hereditary aspects and overview of colorectal tumorigenesis. Brit Med Bull 2002; 64: 27-43.

7. Paulos Gómez A, Ladra González MJ, Álvarez Seoane R, Balado Gómez M, Iglesias Corral J, Conde Freire R, et al. Poliposis gástrica en PAF. Rev Esp Enferm Dig 2007; 99: 545-6.

8. Pinto-Prades JL, Puig-Junoy J, Ortún V. Análisis coste utilidad. Aten Primaria 2001; 27: 569-73.

9. Rubio-Terrés C. Introducción a la utilización de los modelos de Markov en el análisis farmaeconómico. Farm Hosp 2000; 24: 241 7.

10. Bapat B, Noorani H, Cohen Z, Berk T, Mitri A, Gallie B, et al. Cost comparison of predictive genetic testing versus conventional clinical screening for familial adenomatous polyposis. Gut 1999; 44: 698-703.

11. Chikhaoui Y, Gelinas H, Joseph L, Lance JM. Cost-minimization analysis of genetic testing versus clinical screening of at-risk relatives for familiar adenomatous polyposis. Int J Technol Assess Health Care 2002; 18: 67-80. 
12. Cromwell DM, Moore RD, Brensinger JD. Cost-analysis of alternative approaches to colorectal screening in familial adenomatous polyposis. Gastroenterology 1998; 114: 893-901.

13. Ness RM, Holmes AM, Klein R, Dittus R. Cost-utility of one-time colonoscopic screening for colorectal cancer at various ages. Am J Gastroenterol 2000; 95: 1800-11.

14. Ramsey SD, Burke W, Pinsky L, Clarke L, Newcomb P, Khoury MJ. Family history assessment to detect increased risk for colorectal cancer: conceptual considerations and a preliminary economic analysis. Cancer Epidemiol Biomarkers Prev 2005; 14: 2494-500.

15. Graziano A, Gutiérrez A. Poliposis adenomatosa familiar. Disponible en: http://www.proctosite.com (consultado el 22 de marzo de 2008).

16. Bussey HJR. Familial polyposis coli. Family studies, histopathology, differential diagnosis and results of treatment. Baltimore: The Johns Hopkins University Press; 1975.

17. Sánchez R, de Oca J, Parés D, Martí J, Biondo S, Osorio A, et al. Morbilidad y resultados funcionales a largo plazo de los reservorios ileoanales. Cir Esp 2002; 72: 132-6.

18. Pignone M, Saha S, Hoerger T, Mandelblatt J. Cost-effectiveness analyses of colorectal cancer screening: a systematic review for U.S. Preventive services Task Force. Ann Intern Med 2002; 137: 96-104.

19. Moreno A, Vargas E, Soto J, Rejas J. Análisis coste-efectividad del empleo de celecoxib en el tratamiento de la artrosis. Gac Sanit 2003; 17: 27-36.
20. Orden 14 octubre de 2005. BOJA n ${ }^{\circ} 210$. Publicado el 27 de octubre del 2005. p. 46-83.

21. Defunciones según la causa de muerte. Disponible en: www.ine.es (consultado el 12 de enero de 2008).

22. Fernández-Suárez A, Cordero Fernández C, García Lozano R, Pizarro A, Garzón M, Núñez Roldán A. Implicaciones clínicas y éticas del consejo genético en la poliposis adenomatosa familiar. Rev Esp Enferm Dig 2005; 97: 654-65.

23. Half EE, Bresalier RS. Clinical management of hereditary colorectal cancer syndromes. Curr Opin Gastroenterol 2004; 20: 3242.

24. Seeff LC, Manninen DL, Dong FB, Chattopadhyay SK, Nadel MR, Tangka FKL, et al. Is there endoscopic capacity to provide colorectal cancer screening to the unscreened population of the United States? Gastroenterology 2004; 127: 1661-9.

25. Rogowski W. Genetic screening by DNA technology: a systematic review of health economic evidence. Int $\mathrm{J}$ Technol Assess Health Care 2006; 22: 327-37.

26. Rees G, Martin PR, Macrae FA. Screening participation in individuals with a family history of colorectal cancer: a review. Eur J Cancer Care 2007; doi:10.1111/j.1365-2354.2007.00834.x.

27. Cordero Fernández C, Pizarro Moreno A, Garzón Benavides M, García Lozano R, Belda Laguna O, Sobrino S, et al. Seguimiento de los pacientes con poliposis adenomatosa familiar: resultados en una población del sur de España. Rev Esp Enferm Dig 2007; 99: $440-5$. 